\title{
HIGH RESOLUTION OBSERVATIONS OF UMBRAL FINE STRUCTURE
}

\author{
M.SOBOTKA ${ }^{1,2}, \mathrm{~J}^{\text {A BONET }}{ }^{1}, \mathrm{M}^{\text {VAZQUEZ }}{ }^{1}$ \\ (1)Instituto de Astrofísica de Canarias $38200 \mathrm{La}$ Laguna, Spain \\ (2) Astronomical Institute,CS-25165,Ondřejov, Czechoslovakia
}

\begin{abstract}
The main results of a photometric and spectroscopic study of umbral fine structures are presented. The observations were obtained at the SVST ( La Palma) under conditions of very good seeing
\end{abstract}

\section{INTRODUCTION}

In many cases, umbrae are composed of several umbral cores separated by broad light bridges. From the phenomenological point of view, umbral cores consist of a dark background with variable intensity. Well-distinguished local intensity minima in the background are known as dark nuclei (hereafter, DN). The second component of the umbral structure are bright features (BF), including umbral dots or clusters of them (UD), and narrow light bridges (LB). The concepts of "dark" and "bright" have a local meaning only (Sobotka, Bonet, \& Vázquez 1992), because UD in one part of the umbra may show lower intensity than the background in another part.

Umbral dots are observed as tiny bright features, embedded in the dark background. Numerous exciting questions are still open about their brightness, size, and spatial distribution.

In this paper we present a study based on very high spatial resolution of different umbrae belonging to sunspots with different sizes, and at distinct stages of evolution, allowing us for the first time to investigate how the different parameters defining the bright fine features, change with the size, brightness and evolutionary phase of the umbra.

\section{OBSERVATIONS AND DATA ANALYSIS}

During an observing run from 1 to 10 July at the Swedish Vacuum Solar Telescope (SVST) at La Palma, we obtained more than 1000 CCD spectra (line Fe I $5434.5 \AA$ ) with simultaneous CCD slit-jaw images (bandwidth $\lambda 5425 \pm 50$ $\AA$ ). We selected the 18 best couples of spectra and slit-jaw pictures, both with resolution limit of 0.3 ". The slit-jaw images were restored to remove the instrumental effects. The selected frames intercept 7 sunspots, differing in brightness,size, morphology and phase of evolution. Some of them display complex umbrae split into several umbral cores (13 cores in total). In the slitjaw images we identified $35 \mathrm{DN}, 1507 \mathrm{UD}$ and 117 bright grains of faint light bridges. The spectroscopic observations include $10 \mathrm{UD}, 3 \mathrm{DN}$ and 1 bright grain of a LB. 


\section{PHOTOMETRY RESULTS}

From the analysis of restored slit-jaw images we obtained the following results:

1. Good correlation was found between the minimum intensity (ranging between $0.05-0.30$ ) and the mean background intensity $\left(\widetilde{I}_{b a c k}\right.$, ranging between $0.13-0.39$ ) of umbral cores.

2. Small umbral cores $\left(r_{u}<5^{n}\right)$ are on average brighter than large ones.

3 . The average size of dark nuclei (1.5") is similar to the diameter of photospheric granules.

4. The observed peak intensity of UD varies in a broad range from 0.08 to 0.90 . It is strongly related to the intensity of the surrounding background. The same relation is observed in the case of mean values of the peak and background intensities in umbral cores (Figure 1).

5. The observed size of UD $\left(0.43^{n} \pm 0.10\right)$ is independent of the umbral core brightness (Figure 1).

6. Umbral dots are distributed non-uniformly in umbral cores. They are often aligned or clustered around dark nuclei, but are rarely found inside the nuclei.

7. The mean nearest neighbor distance of UD $\left(0.5-0.75^{»}\right)$ decreases with increasing $\bar{I}_{\text {back }}$ of umbral cores (Figure 2). The numerical density of UD increases with increasing $\bar{I}_{b a c k}$.

8. The observed filling factor of UD is not very sensitive to the brightness of umbral cores ( 6 to $8 \%$ in dark umbral cores and 10 to $15 \%$ in the bright ones - see Figure 2).

9. The mean observed size of LB grains ( 0.47 ") is only slightly higher than that of UD. The mean peak-to-peak distance between LB grains is 0.53 ", similar to the minimum value of the nearest neighbor distance of UD.

10. The fractional area of bright grains (0.5) is similar to that of granules in the undisturbed photosphere.

\section{SPECTROSCOPY RESULTS}

The MTF of the telescope (and blurring as well) redistributes part of the intensity coming from the BF to its environment and, on the other hand, part of the intensity of the environment is redistributed to the observed field. Hence, the observed profile in the spectrum is a mixture of the profiles from the $B F$ and from its dark surroundings. This profile can be inverted to a simple semi-empirical two-component model of photospheric layers, described by three parameters: $\Delta \theta_{B F}, \Delta \theta_{E N}$ and a weighting factor. The temperature parameters $\Delta \theta_{B F}$ and $\Delta \theta_{E N}$ define the individual models of the BF and of its environment, respectively. The models are computed by down-scaling $\left(\theta(\tau)=\theta_{\text {ref }}(\tau)+\Delta \theta\right)$ the reference temperature distribution of the undisturbed photosphere, taken from the model of Maltby et al. (1986).

Dark nuclei are assumed to be large enough to allow us to observe their "pure" radiation. Thus, a single-component model is sufficient. In this case we can use a more refined version of the inversion code, allowing a variability of the temperature scaling parameter with optical depth (see Sobotka 1989). 


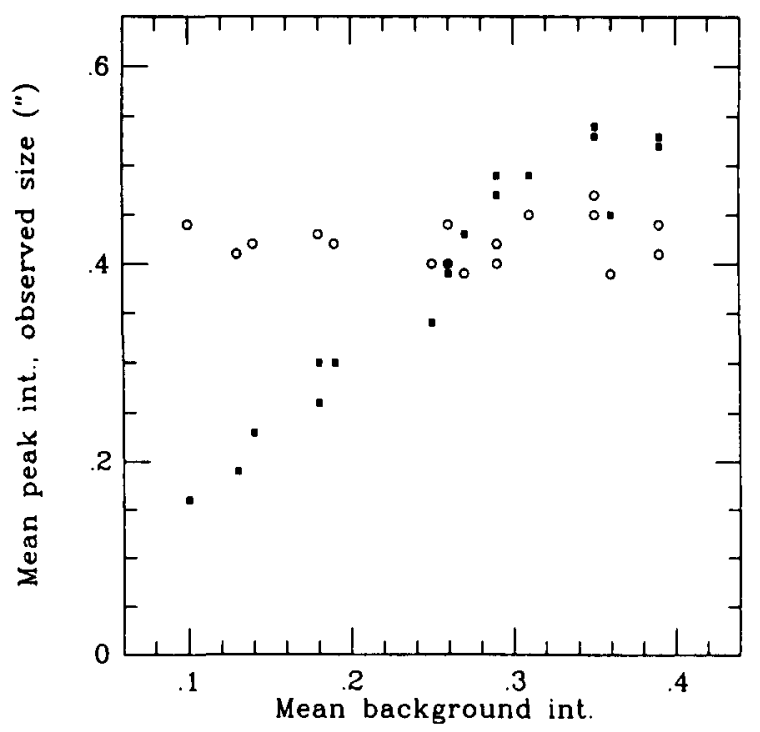

Fig 1.-Umbral dots: Mean values of peak intensity (filled squares) and of observed sizes (circles) in umbral cores versus the mean background intensity of umbral cores



Fig 2.-Umbral dots: Filling factor (circles) and mean nearest neighbor distance (filled squares) in umbral cores versus the mean background intensity of the umbral cores 
Figure 3 illustrates some results of modelling dark (NOAA 6695) and medium-bright (NOAA 6711) umbrae.

From the local two-component models we estimated the "true" intensities of UD and adjacent background. Knowing the approximate "true" intensities, we can easily derive the "true" sizes of $U D$, since the radiative flux is conserved. Eventually, we computed the "real" values of the filling factor.

The results obtained from the analysis of the spectra can be summarized as follows:

1. On average, the estimated "true" intensities are higher than the observed ones only by a factor of about 1.5 .

2. The mean "true" ratio of the UD peak intensities to the adjacent background intensities is $3.0 \pm 0.5$.

3. The typical "true" size of UD is $180-300 \mathrm{~km}(0.25-0.41$ ") and it is uncorrelated to the brightness of UD.

4. The "true" filling factor of UD ranges from 3 to $5 \%$ in dark umbral cores and from 5 to $10 \%$ in bright ones.
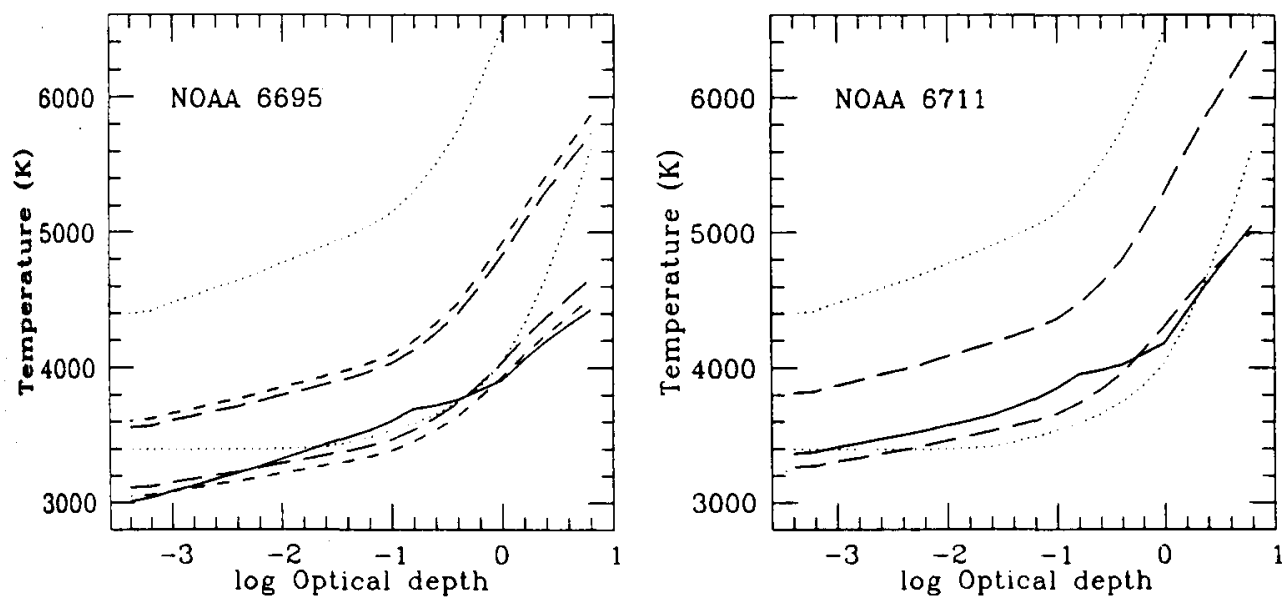

Fig.3.-Simplified temperature stratification in a large dark umbra (NOAA 6695 ) and in a medium-bright one (NOAA 6711). The solid line represents single-component models of DN; Dotted lines give -umbra (model M) and reference photosphere (Maltby et al.,1986); Dashed lines show local twocomponent models of UD and their dark surroundings

\section{References}

Maltby P.,Avrett E.H.,Carlsson M.,Kjeldseth-Moe O., Kurucz R.L., and Loeser R.:1986, Ap. J., , 306,284

Sobotka M.: 1989, Solar Phys., 124,37

Sobotka M.,Bonet J.A.,and Vázquez M.,:1992, Astr. Ap., , 260,437 